\title{
CHARACTERIZING GAUSSIAN FLOWS ARISING FROM ITŌ'S STOCHASTIC DIFFERENTIAL EQUATIONS
}

\author{
SUPRIO BHAR
}

\begin{abstract}
We introduce and characterize a class of flows, which turn out to be Gaussian. This characterization allows us to show, using the Monotonicity inequality, that the transpose of the flow, for an extended class of initial conditions, is the unique solution of the SPDE introduced in Rajeev and Thangavelu (2008).
\end{abstract}

\section{INTRODUCTION}

Itō's stochastic differential equations provide a concrete model for stochastic flows, on which topic there is a considerable literature (see 1, 13, 4, 5, 6, 9, 12, 13, 14, 15, 16, 21, 23 and the references therein). In this paper, we study three interrelated properties of Gaussian flows arising as solutions of stochastic differential equations, viz.

$$
d X_{t}=\sigma\left(X_{t}\right) d B_{t}+b\left(X_{t}\right) d t, t \geq 0 .
$$

Let $L, A, L^{*}, A^{*}, X_{t}, Y_{t}$ be as in [19], with $r$ - the dimension of the Brownian motion there - equal to $d$. Let $\mathcal{S}\left(\mathbb{R}^{d}\right)$ be the space of real valued rapidly decreasing smooth functions on $\mathbb{R}^{d}$. Let $\mathcal{S}^{\prime}\left(\mathbb{R}^{d}\right), \mathcal{E}^{\prime}\left(\mathbb{R}^{d}\right)$ denote the space of tempered distributions on $\mathbb{R}^{d}$ and the space of compactly supported distributions on $\mathbb{R}^{d}$, respectively. Note that, for $\psi \in \mathcal{E}^{\prime}\left(\mathbb{R}^{d}\right),\left\{Y_{t}(\psi)\right\}$ satisfies [19, equation (3.7)]: a.s.

$$
Y_{t}(\psi)=\psi+\int_{0}^{t} A^{*}\left(Y_{s}(\psi)\right) \cdot d B_{s}+\int_{0}^{t} L^{*}\left(Y_{s}(\psi)\right) d s, \forall t \geq 0
$$

in some Hermite Sobolev space $\mathcal{S}_{p}\left(\mathbb{R}^{d}\right)$.

Firstly, we show that the Gaussian flows for

(i) $\sigma$ is a real square matrix of order $d$,

(ii) $b(x):=\alpha+C x, \forall x \in \mathbb{R}^{d}$ where $\alpha=\left(\alpha_{1}, \cdots, \alpha_{d}\right) \in \mathbb{R}^{d}$ and $C=\left(c_{i j}\right)$ is a real square matrix of order $d$,

correspond to those flows that depend 'deterministically' on the initial condition (see Definition 2.1). In particular, $\left\{X_{t}^{0}+e^{t C} x\right\}$ solves (1.1), where $\left\{X_{t}^{x}\right\}$ denotes the solution of (1.1) with initial condition $X_{0}=x$ (see Lemma 3.1).

Secondly, as a consequence the map $x \mapsto \psi\left(X_{t}^{x}\right), \psi \in \mathcal{S}\left(\mathbb{R}^{d}\right)$ is in $\mathcal{S}\left(\mathbb{R}^{d}\right)$. The general question as to when this map (which is $C^{\infty}$ when $\sigma, b$ are smooth) is in $\mathcal{S}\left(\mathbb{R}^{d}\right)$ is to our knowledge, open. This property allows us to extend the map $Y_{t}=X_{t}^{*}$ defined on $\mathcal{E}^{\prime}\left(\mathbb{R}^{d}\right)$ (see [19, equation (3.3)]), from $\mathcal{E}^{\prime}\left(\mathbb{R}^{d}\right) \cap \mathcal{L}^{1}\left(\mathbb{R}^{d}\right) \rightarrow \mathcal{E}^{\prime}\left(\mathbb{R}^{d}\right)$ to

2010 Mathematics Subject Classification. Primary: 60G15, 60H10, 60H15; Secondary: 35K15, $60 \mathrm{H} 30$.

Key words and phrases. Gaussian flows, Stochastic flows, Diffusion processes, Stochastic differential equations, Forward equations, Monotonicity inequality. 
$\mathcal{L}^{1}\left(\mathbb{R}^{d}\right) \rightarrow \mathcal{S}^{\prime}\left(\mathbb{R}^{d}\right)$ and that the processes $\left\{Y_{t}(\psi)\right\}, \psi \in \mathcal{L}^{1}\left(\mathbb{R}^{d}\right)$ satisfy the stochastic partial differential equation [19, (3.7)]. Taking expectation on both sides of this equation, we obtain the existence of solution of the Cauchy problem for $L^{*}$, viz.

$$
\frac{d \psi(t)}{d t}=L^{*} \psi(t) ; \quad \psi(0)=\psi
$$

Thirdly, we note that these flows are unique solutions of [19, equation (3.7)]. The uniqueness of solutions of these equations remains unresolved to date, to our knowledge. As observed in [19, the uniqueness follows from the Monotonicity inequality. This was proved for the pair of operators $\left(A^{*}, L^{*}\right)$ corresponding to Gaussian flows in our class, in [2, Theorem 4.2 and Theorem 4.6]. The same techniques also prove the uniqueness of the Cauchy problem for $L^{*}$.

In Section 2 of this paper we introduce the notion of flows depending deterministically on the initial condition (see Definition 2.1) and prove characterization results (Theorem 2.3, Proposition 2.4). The results say that these type of diffusions correspond to the case when $\sigma$ is a constant and $b$ is in an affine form.

In Section 3, using the composition $x \mapsto \phi\left(X_{t}^{x}\right), \phi \in \mathcal{S}\left(\mathbb{R}^{d}\right)$ we define $\left\{Y_{t}(\psi)\right\}, \psi \in$ $\mathcal{L}^{1}\left(\mathbb{R}^{d}\right)$ and using Monotonicity inequality (see Theorem 3.6) show that it is the unique solution of a stochastic partial differential equation (see Theorem 3.8). As an application of this result, we show $\psi(t)=\mathbb{E} Y_{t}(\psi)$ is the unique solution to (1.2) with initial condition $\psi \in \mathcal{L}^{1}\left(\mathbb{R}^{d}\right)$ (see Theorem 3.9).

In Proposition 3.11, we prove that the tempered distribution $Y_{t}(\psi)$ is given by the integrable function $e^{-t \operatorname{tr}(C)} \tau_{X(t, 0)} \psi\left(e^{-t C}\right.$.) where $\operatorname{tr}(C)$ is the trace of the matrix $C$. This representation is similar to that obtained in [18, Lemma 3.6] for the solution of stochastic differential equations given by certain non-linear operators.

\section{Characterizing DifFusions With the General SOlution DePending DETERMINISTICALLY ON THE INITIAL CONDITION}

In this section, we introduce a class of diffusions and prove some characterization results related to the flow of the diffusions. In particular, these flows turn out to be Gaussian flows. Let $\left(\Omega, \mathcal{F},\left(\mathcal{F}_{t}\right), P\right)$ be a filtered complete probability space satisfying the usual conditions. Let $\left\{B_{t}\right\}$ be the standard $d$ dimensional $\left(\mathcal{F}_{t}\right)$ Brownian motion. Now consider the diffusion:

$$
d X_{t}=\sigma\left(X_{t}\right) d B_{t}+b\left(X_{t}\right) d t, \forall t \geq 0,
$$

where the coefficients $\sigma=\left(\sigma_{i j}\right), b=\left(b_{i}\right), 1 \leq i, j \leq d$ are Lipschitz continuous. Note that $\sigma, b$ satisfy a linear growth condition, i.e. there exists a constant $K>0$ such that

$$
|\sigma(x)|+|b(x)| \leq K(1+|x|), \forall x \in \mathbb{R}^{d},
$$

where $|\cdot|$ denotes the Euclidean norm in the appropriate spaces. For any $x \in \mathbb{R}^{d}$, let $\left\{X_{t}^{x}\right\}$ denote the solution of (2.1) with $X_{0}=x$. We make a definition of the class of diffusions which we are interested in.

Definition 2.1. We say the general solution of the diffusion (2.1) depends deterministically on the initial condition, if there exists a function $f:[0, \infty) \times \mathbb{R}^{d} \rightarrow \mathbb{R}^{d}$ such that for any $x \in \mathbb{R}^{d}$, we have a.s.

$$
X_{t}^{x}(\omega)=f(t, x)+X_{t}^{0}(\omega), t \geq 0 .
$$


Due to the linear growth of the coefficients $\sigma, b$, the first and second moments of $X_{t}^{x}$ exist for all $x, t$. If (2.2) is satisfied, then for all $x, t$ we have

$$
f(t, x)=\mathbb{E} X_{t}^{x}-\mathbb{E} X_{t}^{0}=x+\int_{0}^{t}\left[\mathbb{E} b\left(X_{s}^{x}\right)-\mathbb{E} b\left(X_{s}^{0}\right)\right] d s .
$$

This observation gives the next result. The component functions of $f$ are denoted by $f_{1}, \cdots, f_{d}$.

Lemma 2.2. If the general solution of the diffusion (2.1) depends deterministically on the initial condition, then for all $(t, x) \in[0, \infty) \times \mathbb{R}^{d}$, the partial derivative $\frac{\partial f}{\partial t}(t, x)$ exists and for every fixed $x \in \mathbb{R}^{d}$, the map $t \mapsto \frac{\partial f}{\partial t}(t, x)$ is continuous, where $\frac{\partial f}{\partial t}(t, x)=\left(\frac{\partial f_{1}}{\partial t}(t, x), \cdots, \frac{\partial f_{d}}{\partial t}(t, x)\right)$.

In Theorem 2.3 and Proposition 2.4 we characterize diffusions depending deterministically on the initial condition. In the first result we obtain the characterization under a non-degeneracy condition on $\sigma$ and smoothness assumptions on certain derivatives of $b$ and $f$ and in the second, we consider the case when $f$ is in a product form.

Theorem 2.3. Let $\sigma, b$ be Lipschitz continuous functions. Suppose the following happen:

(i) there exists an $x \in \mathbb{R}^{d}$ such that the determinant of $\left(\sigma_{i j}(x)\right)$ is not zero,

(ii) $b_{i} \in C^{2}\left(\mathbb{R}^{d}, \mathbb{R}\right), i=1, \cdots, d$ where $b=\left(b_{1}, \cdots, b_{d}\right)$,

(iii) for every fixed $x \in \mathbb{R}^{d}$, the map $t \in[0, \infty) \mapsto \frac{\partial f}{\partial t}(t, x)$ is of bounded variation.

Then the general solution of the diffusion (2.1) depends deterministically on the initial condition through (2.2) if and only if $\sigma$ is a real non-singular matrix of order $d$ and $b$ is of the form $b(x)=\alpha+C x$ and $f(t, x)=e^{t C} x$ where $\alpha \in \mathbb{R}^{d}$ and $C$ is a real square matrix of order $d$.

Proof. First we prove the necessity part. For any $x \in \mathbb{R}^{d}$, a.s. $t \geq 0$

$$
\begin{aligned}
f(t, x) & =X_{t}^{x}-X_{t}^{0} \\
& =f(0, x)+\int_{0}^{t}\left[\sigma\left(X_{s}^{x}\right)-\sigma\left(X_{s}^{0}\right)\right] \cdot d B_{s}+\int_{0}^{t}\left[b\left(X_{s}^{x}\right)-b\left(X_{s}^{0}\right)\right] d s .
\end{aligned}
$$

Note that necessarily we must have $f(0, x)=x$. Now rewriting above relation

$$
\int_{0}^{t}\left[\sigma\left(X_{s}^{x}\right)-\sigma\left(X_{s}^{0}\right)\right] \cdot d B_{s}+\int_{0}^{t}\left[b\left(X_{s}^{x}\right)-b\left(X_{s}^{0}\right)-\frac{\partial f}{\partial t}(s, x)\right] d s=0 .
$$

Both the terms are 0 a.s. (see [17, Chapter III, Theorem 12]). By looking at the continuous integrand of the quadratic variation of the martingale, we have for all $x \in \mathbb{R}^{d}, i, j=1, \cdots, d$ a.s. $\left[\sigma_{i j}\left(X_{t}^{x}\right)-\sigma_{i j}\left(X_{t}^{0}\right)\right]^{2}=0, \forall t \geq 0$. Putting $t=0$ we have $\sigma_{i j}(x)=\sigma_{i j}(0), x \in \mathbb{R}^{d}$ i.e. $\sigma$ is a constant $d \times d$ matrix and by our assumption, its determinant is non-zero.

On the other hand, for each $x \in \mathbb{R}^{d}$, a.s. $t \geq 0$,

$$
b\left(X_{t}^{x}\right)-b\left(X_{t}^{0}\right)-\frac{\partial f}{\partial t}(t, x)=0 .
$$

Evaluating at $t=0$ yields $b_{i}(x)=b_{i}(0)+\frac{\partial f_{i}}{\partial t}(0, x), i=1, \cdots, d$. Let $\left\{B_{t}^{(i)}\right\}$ denote the $i$ th component of $\left\{B_{t}\right\}$. Since $b_{i} \in C^{2}\left(\mathbb{R}^{d}, \mathbb{R}\right)$, by Itō formula and equation 
(2.3), we have

$$
\begin{aligned}
\frac{\partial f_{i}}{\partial t}(t, x)-\frac{\partial f_{i}}{\partial t}(0, x) & =\sum_{j, k=1}^{d} \int_{0}^{t}\left(\partial_{j} b_{i}\left(X_{s}^{x}\right)-\partial_{j} b_{i}\left(X_{s}^{0}\right)\right) \sigma_{j k} d B_{s}^{(k)} \\
& +\sum_{j=1}^{d} \int_{0}^{t}\left[\partial_{j} b_{i}\left(X_{s}^{x}\right) b_{j}\left(X_{s}^{x}\right)-\partial_{j} b_{i}\left(X_{s}^{0}\right) b_{j}\left(X_{s}^{0}\right)\right] d s \\
& +\frac{1}{2} \sum_{j, k=1}^{d} \int_{0}^{t}\left(\sigma \sigma^{t}\right)_{j k}\left[\partial_{j} \partial_{k} b_{i}\left(X_{s}^{x}\right)-\partial_{j} \partial_{k} b_{i}\left(X_{s}^{0}\right)\right] d s
\end{aligned}
$$

Again, the martingale term must be zero and therefore so is its quadratic variation. Then for any $i, k=1, \cdots, d$ we have a.s. $\sum_{j=1}^{d} \sigma_{j k}^{2}\left(\partial_{j} b_{i}\left(X_{t}^{x}\right)-\partial_{j} b_{i}\left(X_{t}^{0}\right)\right)^{2}=0, t \geq$ 0 . Evaluating at $t=0$ and simplifying we have $\sum_{j=1}^{d} \sigma_{j k}\left(\partial_{j} b_{i}(x)-\partial_{j} b_{i}(0)\right)=0$. The last equation we can write as

$$
\sigma^{t}\left(\begin{array}{c}
\partial_{1} b_{i}(x)-\partial_{1} b_{i}(0) \\
\cdots \\
\partial_{d} b_{i}(x)-\partial_{d} b_{i}(0)
\end{array}\right)=0 .
$$

Since $\sigma$ is non-singular, for each $i, j=1, \cdots, d$ the function $x \mapsto \partial_{j} b_{i}(x)$ is a constant function. Define $c_{i j}:=\partial_{j} b_{i}(0)$ and write $C=\left(c_{i j}\right)$. Then

$$
\begin{aligned}
b_{i}(x)-b_{i}(0) & =\int_{0}^{x_{1}} \partial_{1} b_{i}\left(y, x_{2}, \cdots, x_{d}\right) d y+\int_{0}^{x_{2}} \partial_{1} b_{i}\left(0, y, x_{3}, \cdots, x_{d}\right) d y \\
& +\cdots+\int_{0}^{x_{d}} \partial_{d} b_{i}(0, \cdots, 0, y) d y=\sum_{j=1}^{d} c_{i j} x_{j}
\end{aligned}
$$

Now for any fixed $x \in \mathbb{R}^{d}$, we have $f(0, x)=x$ and

$$
f(t, x)-x=\int_{0}^{t}\left[b\left(X_{s}^{x}\right)-b\left(X_{s}^{0}\right)\right] d s=\int_{0}^{t} C\left[X_{s}^{x}-X_{s}^{0}\right] d s=\int_{0}^{t} C f(s, x) d s .
$$

Since for each $x$, the map $t \mapsto f(t, x)$ is continuous, we have $f(t, x)=e^{t C} x$. It is easy to check that for above $\sigma, b, f$ the finite variation term in (2.4) vanishes. The converse part can be verified through direct computation.

In Definition 2.1 if the function $f$ is in a product form, then a similar characterization can be obtained without additional smoothness assumptions on $b, f$.

Proposition 2.4. Let $\sigma, b$ be Lipschitz continuous functions.

(i) Suppose the general solution of the diffusion (2.1) depends deterministically on the initial condition, where the function $f$ has the decomposition $f(t, x)=g(t) h(x)$ with $g \in C^{1}([0, \infty), \mathbb{R}), h: \mathbb{R}^{d} \rightarrow \mathbb{R}^{d}$. Then $f(t, x)=\tilde{g}(t) x$ for some $\tilde{g} \in \mathscr{D}$ where

$$
\mathscr{D}:=\left\{g \in C^{1}([0, \infty), \mathbb{R}): g(0)=1\right\} .
$$

(ii) The solution to (2.1) depends deterministically on the initial condition in the following form: there exists $g \in \mathscr{D}$ such that for each $x \in \mathbb{R}^{d}$, a.s. $t \geq 0$

$$
X_{t}^{x}=g(t) x+X_{t}^{0},
$$


if and only if $\sigma$ is a constant $d \times d$ matrix, $b(x)=\alpha+\beta x$ and $g(t)=e^{\beta t}, t \geq$ 0 where $\alpha \in \mathbb{R}^{d}, \beta \in \mathbb{R}$. In this case, the solution has the form

$$
X_{t}^{x}=\left\{\begin{array}{l}
e^{\beta t} x+\sigma \int_{0}^{t} e^{\beta(t-s)} d B_{s}+\frac{e^{\beta t}-1}{\beta} \alpha, \text { if } \beta \neq 0 \\
x+t \alpha+\sigma B_{t}, \text { if } \beta=0 .
\end{array}\right.
$$

Proof. Since a.s. $X_{0}^{x}=f(0, x)+X_{0}^{0}$, we have $x=g(0) h(x), \forall x \in \mathbb{R}^{d}$. So $g(0) \neq 0$. Without loss of generality, we may assume $g(0)=1$. Then $h(x)=x, f(t, x)=g(t) x$. This proves part (i).

If (2.5) holds for some $g \in \mathscr{D}$, then as in Theorem 2.3. we can show $\sigma$ is a constant $d \times d$ matrix. Using (2.3), we have for all $x \in \mathbb{R}^{d}$

$$
\text { a.s. } \quad b\left(X_{t}^{x}\right)-b\left(X_{t}^{0}\right)-g^{\prime}(t) x=0, \forall t \geq 0 .
$$

Putting $t=0$ we have for all $x \in \mathbb{R}^{d}, b(x)=b(0)+g^{\prime}(0) x$. So $b$ is determined by the values $b(0), g^{\prime}(0)$. Using this affine form of $b$ in (2.6) we get the differential equation

$$
g^{\prime}(0) g(t)=g^{\prime}(t) ; t \geq 0 ; \quad g(0)=1 .
$$

The solution is given by $g(t)=e^{g^{\prime}(0) t}, t \geq 0$.

The converse part can be verified through direct computation.

In dimension $d=1$, for convex functions we can apply the following generalization of Itō formula.

Theorem 2.5 ([20, Chapter VI, (1.1) Theorem]). If $\left\{X_{t}\right\}$ is a continuous real valued semimartingale and $f: \mathbb{R} \rightarrow \mathbb{R}$ is a convex function, then there exists a continuous increasing process $\left\{A_{t}^{f}\right\}$ such that a.s. $t \geq 0$

$$
f\left(X_{t}\right)=f\left(X_{0}\right)+\int_{0}^{t} f_{-}^{\prime}\left(X_{s}\right) d X_{s}+\frac{1}{2} A_{t}^{f}
$$

where $f_{-}^{\prime}$ is the left-hand derivative of $f$.

Using the previous theorem, we get the following version of Theorem 2.3

Proposition 2.6. Let $\sigma, b$ be Lipschitz continuous functions on $\mathbb{R}$. Suppose the following happen:

(i) there exists an $x \in \mathbb{R}$ such that $\sigma(x)$ is not zero,

(ii) $b$ is continuously differentiable and is a finite linear combination of convex functions,

(iii) for every fixed $x \in \mathbb{R}^{d}$, the map $t \in[0, \infty) \mapsto \frac{\partial f}{\partial t}(t, x)$ is of bounded variation.

Then the general solution of the diffusion (2.1) depends deterministically on the initial condition through (2.2) if and only if $\sigma$ is a non-zero constant function and $b$ is of the form $b(x)=\alpha+C x$ and $f(t, x)=e^{t C} x$ where $\alpha, C \in \mathbb{R}$.

In the next proposition, we present an example where Definition 2.1 appears. Given a random field $\left(X_{t}^{x}, x \in \mathbb{R}^{d}, t \geq 0\right)$ in many situations it is reasonable to assume that the field can be decomposed as $X_{t}^{x}=Y_{t}^{x}+Z_{t}$, where $\left\{Y_{t}^{x}\right\}$ is a 'local' component and $\left\{Z_{t}\right\}$ is a global component. We show that under certain conditions the 'local' component has to be deterministic. 
Proposition 2.7. Suppose that $Z_{t}=X_{t}^{0}$ and that for all $x \in \mathbb{R}^{d}$, the field $Y_{t}^{x}=$ $X_{t}^{x}-X_{t}^{0}$ is independent of $Z$. In addition assume that $\left\{X_{t}^{x}\right\}$ solves

$$
d X_{t}=\sigma\left(X_{t}\right) d B_{t}+b\left(X_{t}\right) d t, t \geq 0 ; X_{0}=x
$$

and the sigma-fields generated by the processes $\left\{X_{t}^{0}\right\}$ and $\left\{B_{t}\right\}$ are the same. Then $\left\{Y_{t}^{x}\right\}$ is deterministic.

Proof. Under our hypothesis, $\left\{Y_{t}^{x}\right\}$ is both adapted to the said sigma-field and is independent of it. Hence $\left\{Y_{t}^{x}\right\}$ is deterministic.

Example 2.8. If $\sigma(x)$ is non-degenerate for all $x \in \mathbb{R}^{d}$, the sigma-fields generated by the processes $\left\{X_{t}^{0}\right\}$ and $\left\{B_{t}\right\}$ are the same. We present an example which shows that the local part may not be deterministic, if $\sigma(x)$ is degenerate for some $x$. Consider the stochastic differential equations in dimension one:

$$
d X_{t}=x d B_{t}+\left(\alpha-X_{t}\right) d t ; X_{0}=\frac{x^{2}}{2},
$$

where $\alpha$ is some fixed real number. The solution is given by

$$
X_{t}^{x}=e^{-t} \frac{x^{2}}{2}+x \int_{0}^{t} e^{-(t-s)} d B_{s}-\alpha\left(e^{-t}-1\right),
$$

which is not of the form (2.2), but the flow is Gaussian.

In Proposition 2.7, we can allow $\sigma, b$ to be random, but independent of $\left\{B_{t}\right\}$ and then the conclusion still holds, conditional on the $\sigma$-fields of $\sigma, b$. We take this to be in a product form in the next theorem.

Theorem 2.9. Let $\left(\Omega^{\prime}, \mathcal{F}^{\prime}, P^{\prime}\right)$ be a complete probability space and $\left(\Omega^{\prime \prime}, \mathcal{F}^{\prime \prime},\left(\mathcal{F}_{t}^{\prime \prime}\right), P^{\prime \prime}\right)$ a filtered complete probability space satisfying the usual conditions. Define $\Omega:=$ $\Omega^{\prime} \times \Omega^{\prime \prime}$. Consider the filtered probability space $\left(\Omega, \mathcal{F}^{\prime} \otimes \mathcal{F}^{\prime \prime},\left(\mathcal{F}^{\prime} \otimes \mathcal{F}_{t}^{\prime \prime}\right), P^{\prime} \times P^{\prime \prime}\right)$. Let $\left\{B_{t}\right\}$ be an $\left(\mathcal{F}_{t}^{\prime \prime}\right)$ Brownian motion. Assume that $\mathcal{F}_{t}^{\prime \prime}=\sigma\left\{B_{s}: 0 \leq s \leq t\right\}$ and $\mathcal{F}^{\prime \prime}=\sigma\left\{B_{t}: t \geq 0\right\}$. Let $b: \mathbb{R}^{d} \times \Omega \rightarrow \mathbb{R}^{d}$ be $\mathcal{B}\left(\mathbb{R}^{d}\right) \otimes \mathcal{F}^{\prime} \otimes \mathcal{F}_{0}^{\prime \prime} / \mathcal{B}\left(\mathbb{R}^{d}\right)$ measurable and $\sigma: \mathbb{R}^{d} \times \Omega \rightarrow \mathbb{R}^{d \times d}$ be $\mathcal{B}\left(\mathbb{R}^{d}\right) \otimes \mathcal{F}^{\prime} \otimes \mathcal{F}_{0}^{\prime \prime} / \mathcal{B}\left(\mathbb{R}^{d \times d}\right)$ measurable, where $\mathcal{B}\left(\mathbb{R}^{d}\right)$ denotes the Borel sigma field on $\mathbb{R}^{d}$. Suppose that a unique strong solution to the following stochastic differential equation

$$
d X_{t}=\sigma\left(X_{t}\right) d B_{t}+b\left(X_{t}\right) d t, t \geq 0 ; X_{0}=x
$$

exists for each $x \in \mathbb{R}^{d}$. Denote the solution by $\left\{X_{t}^{x}\right\}$. Suppose that

(i) $\sigma\left\{B_{t}: t \geq 0\right\}=\sigma\left\{X_{t}^{0}: t \geq 0\right\}$,

(ii) $\left\{X_{t}^{x}-X_{t}^{0}: x \in \mathbb{R}^{d}, t \geq 0\right\}$ and $\left\{B_{t}: t \geq 0\right\}$ are independent.

Then a.s. $\omega^{\prime}\left(P^{\prime}\right)$, a.s. $\omega^{\prime \prime}\left(P^{\prime \prime}\right)$ we have the process $\left\{X_{t}^{x}-X_{t}^{0}\right\}$ depends on $\omega^{\prime}$ alone.

Proof. By condition (ii), a.s. $\omega^{\prime}\left(P^{\prime}\right),\left\{B_{t}: t \geq 0\right\}$ and $\left\{X_{t}^{x}\left(\omega^{\prime}, \cdot\right)-X_{t}^{0}\left(\omega^{\prime}, \cdot\right): t \geq\right.$ $\left.0, x \in \mathbb{R}^{d}\right\}$ are independent.

Since $\left\{X_{t}^{x}\right\}$ is the strong solution of a stochastic differential equation, there exists a $P^{\prime}$-null set $\mathcal{N}^{\prime} \subset \Omega^{\prime}$ such that for every $\omega \in \Omega^{\prime} \backslash \mathcal{N}^{\prime}$, a.s. $\omega^{\prime \prime}\left(P^{\prime \prime}\right)$,

$$
X_{t}^{x}\left(\omega^{\prime}, \omega^{\prime \prime}\right)=x+\left(\int_{0}^{t} \sigma\left(X_{s}\right) d B_{s}\right)\left(\omega^{\prime}, \omega^{\prime \prime}\right)+\int_{0}^{t} b\left(X_{s}\left(\omega^{\prime}, \omega^{\prime \prime}\right), \omega^{\prime}, \omega^{\prime \prime}\right) d s, t \geq 0 .
$$

Hence a.s. $\omega^{\prime}\left(P^{\prime}\right)$, the random variables $X_{t}^{x}\left(\omega^{\prime}, \cdot\right), t \geq 0, x \in \mathbb{R}^{d}$ are measurable with respect to $\sigma\left\{B_{t}: t \geq 0\right\}$ and by condition $(i)$, so are $X_{t}^{x}\left(\omega^{\prime}, \cdot\right)-X_{t}^{0}\left(\omega^{\prime}, \cdot\right), t \geq$ 
$0, x \in \mathbb{R}^{d}$.

Hence a.s. $\omega^{\prime}\left(P^{\prime}\right), X_{t}^{x}\left(\omega^{\prime}, \omega^{\prime \prime}\right)-X_{t}^{0}\left(\omega^{\prime}, \omega^{\prime \prime}\right), t \geq 0, x \in \mathbb{R}^{d}$ is deterministic in $\omega^{\prime \prime}$, i.e. the random variables depend on $\omega^{\prime}$ alone.

Remark 2.10. (i) One may formulate and prove similar results for the following type of condition

$$
X_{t}^{s, x}(\omega)=f(t, s, x)+X_{t}^{s, 0}(\omega), t \geq s ; X_{s}^{s, x}=x
$$

for $s \geq 0, x \in \mathbb{R}^{d}$.

(ii) If equation (2.2) holds, then $f(t, x)=\mathbb{E}\left[X_{t}^{x}-X_{t}^{0}\right]$. As such the conditions on $f$ (in Theorem 2.3. Proposition 2.4) can be stated in terms of $\mathbb{E} X_{t}^{x}, x \in \mathbb{R}^{d}$.

(iii) Diffusions satisfying (2.2) also satisfy the following condition: for any $x, y \in$ $\mathbb{R}^{d}$, a.s. $t \geq 0$

$$
X_{t}^{x}-X_{t}^{y}=f(t, x)-f(t, y) .
$$

In certain situations such differences were shown to be diffusions (see [22, Proposition 2.2]).

(iv) Semimartingales with independent increments have been considered in [11, Chapter II]. In particular, it was shown that any rcll process with independent increments must be a sum of a semimartingale with independent increments and a deterministic part ([11, Chapter II, 5.1 Theorem]). This is similar to (2.2), but we are interested in the dependence of a possible deterministic part of the flows (generated by stochastic differential equations) on the initial condition.

\section{Probabilistic representations of the solutions of the Forward EQUATIONS}

Let $\left(\Omega, \mathcal{F},\left(\mathcal{F}_{t}\right), P\right)$ be a filtered complete probability space satisfying the usual conditions and let $\left\{B_{t}\right\}$ denote the standard $d$ dimensional $\left(\mathcal{F}_{t}\right)$ Brownian motion. Let $\mathcal{S}^{\prime}\left(\mathbb{R}^{d}\right)$ be the space of tempered distributions, which is the dual of the space $\mathcal{S}\left(\mathbb{R}^{d}\right)$ of real valued rapidly decreasing smooth functions on $\mathbb{R}^{d}$. Let $\mathcal{L}^{1}\left(\mathbb{R}^{d}\right)$ denote the space of integrable functions. Note that $\mathcal{L}^{1}\left(\mathbb{R}^{d}\right) \subset \mathcal{S}^{\prime}\left(\mathbb{R}^{d}\right)$.

We obtain probabilistic representations of the solutions of (1.2) where

(i) $\sigma$ is a real square matrix of order $d$ and $b(x):=\alpha+C x, \forall x \in \mathbb{R}^{d}$ where $\alpha=\left(\alpha_{1}, \cdots, \alpha_{d}\right) \in \mathbb{R}^{d}$ and $C=\left(c_{i j}\right)$ is a real square matrix of order $d$,

(ii) $\psi \in \mathcal{L}^{1}\left(\mathbb{R}^{d}\right)$.

Since $\sigma, b$ are $C^{\infty}$ functions with bounded derivatives, there exists a diffeomorphic modification of the solution of

$$
d X_{t}=\sigma\left(X_{t}\right) \cdot d B_{t}+b\left(X_{t}\right) d t ; \quad X_{0}=x
$$

(see [14, [19, Theorem 2.1]). We first observe that such a modification can be written in an explicit form, with a null set independent of deterministic initial conditions of (3.1).

Lemma 3.1. Let $\sigma, b$ be as above. Let $\{X(t, 0)\}$ denote the solution of (3.1) with initial condition $X_{0}=0$. Then a.s. for all $t \geq 0, x \in \mathbb{R}^{d}$

$$
X(t, 0)+e^{t C} x=x+\int_{0}^{t} \sigma d B_{s}+\alpha t+\int_{0}^{t} C\left(X(s, 0)+e^{s C} x\right) d s
$$


so that the sum $\left\{X(t, 0)+e^{t C} x\right\}$ solves the stochastic differential equation

$$
d X_{t}=\sigma\left(X_{t}\right) \cdot d B_{t}+b\left(X_{t}\right) d t ; \quad X_{0}=x .
$$

Proof. The proof follows from the observation that $\sigma(X(t, 0))=\sigma\left(X(t, 0)+e^{t C} x\right)=$ $\sigma$ for any $t \geq 0, x \in \mathbb{R}^{d}$.

For the case $\sigma=I d$ ( $I d$ denotes the $d \times d$ identity matrix), $b(x)=-x$, we get the well-known Ornstein-Uhlenbeck diffusion.

Let $\left(\mathcal{S}_{p}\left(\mathbb{R}^{d}\right),\|\cdot\|_{p}\right), p \in \mathbb{R}$ be the Hermite Sobolev spaces (see [10, Chapter 1.3]), which are real separable Hilbert spaces and are completions of $\left(\mathcal{S}\left(\mathbb{R}^{d}\right),\|\cdot\|_{p}\right)$. Given $\psi \in \mathcal{S}\left(\mathbb{R}^{d}\right)\left(\right.$ or $\left.\mathcal{S}_{p}\left(\mathbb{R}^{d}\right)\right)$ and $\phi \in \mathcal{S}^{\prime}\left(\mathbb{R}^{d}\right)$ (or $\mathcal{S}_{-p}\left(\mathbb{R}^{d}\right)$ ), the action of $\phi$ on $\psi$ will be denoted by $\langle\phi, \psi\rangle$. For each $x$, let $\delta_{x}$ denote the Dirac distribution supported at the point $x$. The following result lists some properties of the Dirac distributions.

Proposition 3.2. $\quad$ (i) ([19, Theorem 4.1]) Let $x \in \mathbb{R}^{d}$. Then $\delta_{x} \in \mathcal{S}_{-p}\left(\mathbb{R}^{d}\right)$ for any $p>\frac{d}{4}$. Further if $p>\frac{d}{4}$, then $\lim _{|x| \rightarrow \infty}\left\|\delta_{x}\right\|_{-p}=0$ and there exists a constant $R=R(p)>0$ such that $\left\|\delta_{x}\right\|_{-p} \leq R, \forall x \in \mathbb{R}^{d}$.

(ii) Let $p>\frac{d}{4}$. Then the map $x \in \mathbb{R}^{d} \mapsto \delta_{x} \in \mathcal{S}_{-p}\left(\mathbb{R}^{d}\right)$ is continuous.

Proof. Only part (ii) requires a proof. In [19, Proposition 3.1] it was shown that the map $x \in \mathbb{R}^{d} \mapsto \tau_{x} \phi \in \mathcal{S}_{q}\left(\mathbb{R}^{d}\right)$ is continuous where

(a) $\phi \in \mathcal{S}_{q}\left(\mathbb{R}^{d}\right)$ for some real number $q$,

(b) $\tau_{x}: \mathcal{S}^{\prime}\left(\mathbb{R}^{d}\right) \rightarrow \mathcal{S}^{\prime}\left(\mathbb{R}^{d}\right), x \in \mathbb{R}^{d}$ are translation operators, which on functions act as follows: $\left(\tau_{x} f\right)(y):=f(y-x), y \in \mathbb{R}^{d}$.

But $\delta_{0} \in \mathcal{S}_{-p}\left(\mathbb{R}^{d}\right)$ (by part $(i)$ ) and $\tau_{x} \delta_{0}=\delta_{x}$. Hence the required continuity follows.

Lemma 3.3. Let $p>\frac{d}{4}$. Then $\mathcal{L}^{1}\left(\mathbb{R}^{d}\right) \subset \mathcal{S}_{-p}\left(\mathbb{R}^{d}\right)$.

Proof. For $\psi \in \mathcal{L}^{1}\left(\mathbb{R}^{d}\right), \int_{\mathbb{R}^{d}}|\psi(x)| \cdot\left\|\delta_{x}\right\|_{-p} d x \leq R \int_{\mathbb{R}^{d}}|\psi(x)| d x<\infty$, where $R=$ $R(p)$ is the constant given by Proposition 3.2(i)] Hence $\int_{\mathbb{R}^{d}} \psi(x) \delta_{x} d x$ is a welldefined element of $\mathcal{S}_{-p}\left(\mathbb{R}^{d}\right)$. It can be checked that as a tempered distribution $\psi=\int_{\mathbb{R}^{d}} \psi(x) \delta_{x} d x$ and hence $\psi \in \mathcal{S}_{-p}\left(\mathbb{R}^{d}\right)$.

In what follows, $\{X(t, x)\}$ and $\mathcal{N}$ will denote the solution and the null set mentioned in Lemma 3.1 respectively. As in [19, equation (3.3)]), for any $\psi \in \mathcal{L}^{1}\left(\mathbb{R}^{d}\right)$ we define

$$
Y_{t}(\omega)(\psi):=\int_{\mathbb{R}^{d}} \psi(x) \delta_{X(t, x, \omega)} d x, \omega \in \Omega \backslash \mathcal{N}
$$

and set $Y_{t}(\omega)(\psi):=0$, if $\omega \in \mathcal{N}$.

Proposition 3.4. Let $\psi,\{X(t, x)\},\left\{Y_{t}(\psi\}\right)$ be as above. Let $p>\frac{d}{4}$. Then $\left\{Y_{t}(\psi)\right\}$ is an $\left(\mathcal{F}_{t}\right)$ adapted $\mathcal{S}_{-p}\left(\mathbb{R}^{d}\right)$ valued continuous process. Furthermore, $Y_{t}(\psi)$ is normbounded, where the bound can be chosen to be independent of $t$.

Proof. Since $\psi \in \mathcal{L}^{1}\left(\mathbb{R}^{d}\right)$ and $\delta_{x}, x \in \mathbb{R}^{d}$ are uniformly bounded in $\mathcal{S}_{-p}\left(\mathbb{R}^{d}\right)$ (Proposition 3.2(i),$Y_{t}(\psi)$ is a well-defined element of $\mathcal{S}_{-p}\left(\mathbb{R}^{d}\right)$ for any $p>\frac{d}{4}$. Existence of a bound of $Y_{t}(\psi)$ independent of $t$ follows from the bound on $\delta_{x}, x \in \mathbb{R}^{d}$.

Since $\{X(t, x)\}$ is $\left(\mathcal{F}_{t}\right)$ adapted for each $x$, so is $\left\{Y_{t}(\psi)\right\}$. Since $\{X(t, x)\}$ has continuous paths for each $x \in \mathbb{R}^{d}$, by Proposition 3.2(ii) $\left\{\delta_{X(t, x)}\right\}$ also has continuous paths in $\mathcal{S}_{-p}\left(\mathbb{R}^{d}\right)$ for each $x \in \mathbb{R}^{d}$. Continuity of $\left\{Y_{t}(\psi)\right\}$ follows from the Dominated Convergence theorem. 
For any $t \geq 0, \omega \in \Omega \backslash \mathcal{N}, x \mapsto X(t, x, \omega)$ is affine and hence the map $x \mapsto$ $\phi(X(t, x, \omega))$ is in $\mathcal{S}\left(\mathbb{R}^{d}\right)$ whenever $\phi \in \mathcal{S}\left(\mathbb{R}^{d}\right)$. Consider the linear map $X_{t}(\omega)$ : $\mathcal{S}\left(\mathbb{R}^{d}\right) \rightarrow \mathcal{S}\left(\mathbb{R}^{d}\right)$ defined by $\left(X_{t}(\omega) \phi\right)(x):=\phi(X(t, x, \omega)), x \in \mathbb{R}^{d}$. The following property of the map $X_{t}(\omega)$ will be used in our analysis.

Lemma 3.5. Fix any $t \geq 0, \omega \in \Omega \backslash \mathcal{N}$. The linear map $X_{t}(\omega): \mathcal{S}\left(\mathbb{R}^{d}\right) \rightarrow \mathcal{S}\left(\mathbb{R}^{d}\right)$ is continuous.

Proof. Certain seminorms given by supremums on $\mathbb{R}^{d}$ generates the topology on $\mathcal{S}\left(\mathbb{R}^{d}\right)$ (see [10, Chapter 1.3]). Since $x \mapsto X(t, x, \omega)$ is affine, we can establish necessary estimates in terms of the said seminorms.

Let $X_{t}^{*}(\omega): \mathcal{S}^{\prime}\left(\mathbb{R}^{d}\right) \rightarrow \mathcal{S}^{\prime}\left(\mathbb{R}^{d}\right)$ denote the transpose of the map $X_{t}(\omega)$. Then for any $\theta \in \mathcal{S}^{\prime}\left(\mathbb{R}^{d}\right)$,

$$
\left\langle X_{t}^{*}(\theta), \phi\right\rangle=\left\langle\theta, X_{t}(\phi)\right\rangle, \forall \phi \in \mathcal{S}\left(\mathbb{R}^{d}\right) .
$$

Using (3.3), for any $\phi \in \mathcal{S}\left(\mathbb{R}^{d}\right), \psi \in \mathcal{L}^{1}\left(\mathbb{R}^{d}\right)$ we have

$$
\left\langle Y_{t}(\psi), \phi\right\rangle=\int_{\mathbb{R}^{d}} \psi(x) \phi(X(t, x)) d x=\int_{\mathbb{R}^{d}} \psi(x)\left(X_{t}(\phi)\right)(x) d x=\left\langle\psi, X_{t}(\phi)\right\rangle .
$$

This implies

$$
Y_{t}(\psi)=X_{t}^{*}(\psi)
$$

Consider the derivative maps $\partial_{i}: \mathcal{S}\left(\mathbb{R}^{d}\right) \rightarrow \mathcal{S}\left(\mathbb{R}^{d}\right)$ for $i=1, \cdots, d$ as in [19]. On $\mathcal{S}\left(\mathbb{R}^{d}\right)$ consider the multiplication operators $M_{i}, i=1, \cdots, d$ defined by $\left(M_{i} \phi\right)(x):=$ $x_{i} \phi(x), \phi \in \mathcal{S}\left(\mathbb{R}^{d}\right), x=\left(x_{1}, \cdots, x_{d}\right) \in \mathbb{R}^{d}$. By duality these operators can be extended to $M_{i}: \mathcal{S}^{\prime}\left(\mathbb{R}^{d}\right) \rightarrow \mathcal{S}^{\prime}\left(\mathbb{R}^{d}\right)$.

Let $\left\{e_{1}, \cdots, e_{d}\right\}$ be the standard basis vectors in $\mathbb{R}^{d}$. Let $n=\left(n_{1}, \cdots, n_{d}\right) \in$ $\mathbb{Z}_{+}^{d}:=\left\{n=\left(n_{1}, \cdots, n_{d}\right): n_{i}\right.$ are non-negative integers $\}$. Let $h_{n}, n \in \mathbb{Z}_{+}^{d}$ be the Hermite functions (see [10, Chapter 1.3]). Note that $\left\{(2|n|+d)^{-p} h_{n}: n \in \mathbb{Z}_{+}^{d}\right\}$ is an orthonormal basis for $\mathcal{S}_{p}\left(\mathbb{R}^{d}\right)$, where $|n|=n_{1}+\cdots+n_{d}$. We also have (see [7] Appendix A.5, equation (A.26)])

$$
\partial_{i} h_{n}=\sqrt{\frac{n_{i}}{2}} h_{n-e_{i}}-\sqrt{\frac{n_{i}+1}{2}} h_{n+e_{i}}
$$

and

$$
x_{i} h_{n}(x)=\sqrt{\frac{n_{i}+1}{2}} h_{n+e_{i}}(x)+\sqrt{\frac{n_{i}}{2}} h_{n-e_{i}}(x),
$$

with the convention that for a multi-index $n=\left(n_{1}, \cdots, n_{d}\right)$, if $n_{i}<0$ for some $i$, then $h_{n} \equiv 0$. Above recurrence implies that $\partial_{i}, M_{i}: \mathcal{S}_{p}\left(\mathbb{R}^{d}\right) \rightarrow \mathcal{S}_{p-\frac{1}{2}}\left(\mathbb{R}^{d}\right)$ are bounded linear operators, for any $p \in \mathbb{R}$.

The operators $A, L$ are given as follows: for $\phi \in \mathcal{S}\left(\mathbb{R}^{d}\right)$ and $x \in \mathbb{R}^{d}$,

$$
\left\{\begin{array}{l}
A \phi:=\left(A_{1} \phi, \cdots, A_{r} \phi\right) \\
A_{i} \phi(x):=\sum_{k=1}^{d} \sigma_{k i}(x) \partial_{k} \phi(x), \\
L \phi(x):=\frac{1}{2} \sum_{i, j=1}^{d}\left(\sigma \sigma^{t}\right)_{i j}(x) \partial_{i j}^{2} \phi(x)+\sum_{i=1}^{d} b_{i}(x) \partial_{i} \phi(x),
\end{array}\right.
$$


where $\sigma^{t}$ denotes the transpose of $\sigma$. For $\psi \in \mathcal{S}^{\prime}\left(\mathbb{R}^{d}\right)$ define the adjoint operators $A^{*}, L^{*}$ as follows.

$$
\left\{\begin{array}{l}
A^{*} \psi:=\left(A_{1}^{*} \psi, \cdots, A_{r}^{*} \psi\right), \\
A_{i}^{*} \psi:=-\sum_{k=1}^{d} \partial_{k}\left(\sigma_{k i} \psi\right), \\
L^{*} \psi:=\frac{1}{2} \sum_{i, j=1}^{d} \partial_{i j}^{2}\left(\left(\sigma \sigma^{t}\right)_{i j} \psi\right)-\sum_{i=1}^{d} \partial_{i}\left(b_{i} \psi\right) .
\end{array}\right.
$$

We now look at $A^{*}, L^{*}$ as operators on $\mathcal{S}_{p}\left(\mathbb{R}^{d}\right)$.

Theorem 3.6. Fix $p \in \mathbb{R}$. There exist constants $C_{1}=C_{1}(p), C_{2}=C_{2}(p)>0$ such that

$$
\left\|A_{i}^{*} \theta\right\|_{p-\frac{1}{2}} \leq C_{1}\|\theta\|_{p},\left\|L^{*} \theta\right\|_{p-1} \leq C_{2}\|\theta\|_{p}, \forall \theta \in \mathcal{S}_{p}\left(\mathbb{R}^{d}\right)
$$

Furthermore, we have the Monotonicity inequality for $\left(A^{*}, L^{*}\right)$, i.e. there exists a constant $C_{p}>0$ such that

$$
2\left\langle\theta, L^{*} \theta\right\rangle_{p}+\left\|A^{*} \theta\right\|_{H S(p)}^{2} \leq C_{p}\|\theta\|_{p}^{2}, \forall \theta \in \mathcal{S}_{p+1}\left(\mathbb{R}^{d}\right),
$$

where $\left\|A^{*} \theta\right\|_{H S(p)}^{2}:=\sum_{i=1}^{d}\left\|A_{i}^{*} \theta\right\|_{p}^{2}$.

Proof. For any $q \in \mathbb{R}, \partial_{i}, M_{i}: \mathcal{S}_{q}\left(\mathbb{R}^{d}\right) \rightarrow \mathcal{S}_{q-\frac{1}{2}}\left(\mathbb{R}^{d}\right)$ are bounded linear operators. Using the definitions of $A^{*}$ and $L^{*}$, estimates on the norms follows.

Proof of the Monotonicity inequality for $\left(A^{*}, L^{*}\right)$ follows from [2, Theorem 4.2 and Theorem 4.6].

For $p>\frac{d}{4}$, let $R=R(p)$ be as in Proposition 3.2(i) Then

$$
\mathbb{E}\left\|Y_{t}(\psi)\right\|_{-p}^{2} \leq R^{2}\left(\int_{\mathbb{R}^{d}}|\psi(x)| d x\right)^{2}<\infty
$$

Using the norm estimates on $A_{i}^{*}, i=1, \cdots, d$ in the previous theorem and equation (3.7) the next result can be proved.

Proposition 3.7. Let $p>\frac{d}{4}$. Then $\left\{\int_{0}^{t} A^{*}\left(Y_{s}(\psi)\right) . d B_{s}\right\}$ is an $\left(\mathcal{F}_{t}\right)$ adapted $\mathcal{S}_{-p-\frac{1}{2}}\left(\mathbb{R}^{d}\right)$ valued continuous martingale.

Next result is analogous to [19, Theorem 3.3], except the uniqueness part.

Theorem 3.8. Let $p>\frac{d}{4}$ and $\psi \in \mathcal{L}^{1}\left(\mathbb{R}^{d}\right)$. Then the $\mathcal{S}_{-p}\left(\mathbb{R}^{d}\right)$ valued continuous adapted process $\left\{Y_{t}(\psi)\right\}$ satisfies the following equation in $\mathcal{S}_{-p-1}\left(\mathbb{R}^{d}\right)$, a.s.

$$
Y_{t}(\psi)=\psi+\int_{0}^{t} A^{*}\left(Y_{s}(\psi)\right) \cdot d B_{s}+\int_{0}^{t} L^{*}\left(Y_{s}(\psi)\right) d s, \forall t \geq 0
$$

This solution is also unique.

Proof. By Itō's formula for any $\phi \in \mathcal{S}\left(\mathbb{R}^{d}\right)$, and any $x \in \mathbb{R}^{d}$ we have

$$
\left(X_{t}(\phi)\right)(x)=\phi(x)+\int_{0}^{t}\left(X_{s}(A \phi)\right)(x) \cdot d B_{s}+\int_{0}^{t}\left(X_{s}(L \phi)\right)(x) d s .
$$

Since $L \phi \in \mathcal{S}\left(\mathbb{R}^{d}\right),\left\{x \mapsto\left(X_{t}(L \phi)\right)(x)\right\}$ is an $\mathcal{S}\left(\mathbb{R}^{d}\right)$ valued process. Using differentiation under the sign of integration we can establish the existence of all derivatives of $x \mapsto \int_{0}^{t}\left(X_{s}(L \phi)\right)(x) d s$ for any $t \geq 0$. Given non-negative integers $N, \alpha_{1}, \cdots, \alpha_{d}$, the terms

$$
\sup _{x \in \mathbb{R}^{d}}\left(1+|x|^{2}\right)^{N}\left|\partial_{1}^{\alpha_{1}} \cdots \partial_{d}^{\alpha_{d}}\left(X_{s}(L \phi)\right)(x)\right|, s \in[0, t]
$$


can be dominated uniformly in $s$ and hence $\left\{x \mapsto \int_{0}^{t}\left(X_{s}(L \phi)\right)(x) d s\right\}$ is an $\mathcal{S}\left(\mathbb{R}^{d}\right)$ valued process. From (3.9), we conclude that $\left\{x \mapsto \int_{0}^{t}\left(X_{s}(A \phi)\right)(x) \cdot d B_{s}\right\}$ is an $\mathcal{S}\left(\mathbb{R}^{d}\right)$ valued process, since the other terms are so. Then for $\phi \in \mathcal{S}\left(\mathbb{R}^{d}\right)$, using (3.4) we can show a.s. $t \geq 0$

$$
\left\langle Y_{t}(\psi), \phi\right\rangle=\left\langle\psi+\int_{0}^{t} A^{*} Y_{s}(\psi) \cdot d B_{s}+\int_{0}^{t} L^{*} Y_{s}(\psi) d s, \phi\right\rangle .
$$

The argument for above equality is similar to [19, Theorem 3.3]. This proves $Y_{t}(\psi)$ solves (3.8) in $\mathcal{S}_{-p-1}\left(\mathbb{R}^{d}\right)$.

To prove the uniqueness, let $\left\{Y_{t}^{1}\right\},\left\{Y_{t}^{2}\right\}$ be two solutions of (3.8) and let $Z_{t}:=$ $Y_{t}^{1}-Y_{t}^{2}, t \geq 0$. Then using Itō formula for $\|\cdot\|_{-p-1}^{2}$ we obtain a.s. for all $t \geq 0$

$$
\left\|Z_{t}\right\|_{-p-1}^{2}=\int_{0}^{t}\left[2\left\langle Z_{s}, L^{*} Z_{s}\right\rangle_{-p-1}+\sum_{i=1}^{d}\left\|A_{i}^{*} Z_{s}\right\|_{-p-1}^{2}\right] d s+M_{t}
$$

where $\left\{M_{t}\right\}$ is some continuous local martingale with $M_{0}=0$. Now using the Monotonicity inequality (Theorem 3.6) and the Gronwall's inequality we can show $Z_{t}=0, t \geq 0$ a.s., which shows a.s. $Y_{t}^{1}=Y_{t}^{2}, t \geq 0$. The argument is similar to [18, Lemma 3.6].

We now prove the existence and uniqueness of solution to (1.2) with initial condition $\psi \in \mathcal{L}^{1}\left(\mathbb{R}^{d}\right)$. This result is analogous to [19, Theorems $\left.4.3,4.4\right]$.

Theorem 3.9. Let $p>\frac{d}{4}$ and $\psi \in \mathcal{L}^{1}\left(\mathbb{R}^{d}\right)$. Then $\psi(t):=\mathbb{E} Y_{t}(\psi)$ solves the initial value problem (1.2), i.e.

$$
\mathbb{E} Y_{t}(\psi)=\psi+\int_{0}^{t} L^{*} \mathbb{E} Y_{s}(\psi) d s
$$

holds in $\mathcal{S}_{-p-1}\left(\mathbb{R}^{d}\right)$. Furthermore this is the unique solution.

Proof. The arguments are similar to [19, Theorems 4.3, 4.4]. Taking term by term expectation on both sides of (3.8) and using Proposition 3.7 we can show $\psi(t):=$ $\mathbb{E} Y_{t}(\psi)$ solves $(1.2)$ in $\mathcal{S}_{-p-1}\left(\mathbb{R}^{d}\right)$. Here $L^{*} \mathbb{E} Y_{s}(\psi)=\mathbb{E} L^{*} Y_{s}(\psi)$, since $L^{*}$ is a bounded linear operator from $\mathcal{S}_{-p}\left(\mathbb{R}^{d}\right)$ to $\mathcal{S}_{-p-1}\left(\mathbb{R}^{d}\right)$.

The proof of uniqueness of the solution is same as in [19, Theorem 4.4]. We use the Monotonicity inequality in Theorem 3.6 and the Gronwall's inequality.

Remark 3.10. In [19, Theorem 4.4] the Monotonicity inequality was in the hypothesis, whereas in our case it has been proved.

The process $\left\{Y_{t}(\psi)\right\}$ can also be described in terms of $\{X(t, 0)\}$ without using the integral representation (3.3). We show that the tempered distribution $Y_{t}(\psi)(\omega)$ is given by an integrable function. This representation of $\left\{Y_{t}(\psi)\right\}$ is similar to the representation obtained in [18, Lemma 3.6], where the author looked at the solution of stochastic partial differential equations governed by certain non-linear operators.

Proposition 3.11. Let $\psi \in \mathcal{L}^{1}\left(\mathbb{R}^{d}\right)$ and $\omega \in \mathcal{N}$. Then

$$
Y_{t}(\psi)=e^{-t \operatorname{tr}(C)} \tau_{Z_{t}} \psi_{t}(\cdot),
$$

where $Z_{t}:=X(t, 0), \psi_{t}(x):=\psi\left(e^{-t C} x\right)$ for $t \geq 0, x \in \mathbb{R}^{d}$ and $\operatorname{tr}(C)$ is the trace of the matrix $C$. 
Proof. For any $\phi \in \mathcal{S}\left(\mathbb{R}^{d}\right)$, we have

$$
\begin{aligned}
\left\langle Y_{t}(\psi), \phi\right\rangle & =\int_{\mathbb{R}^{d}} \psi(x) \phi(X(t, x)) d x=\int_{\mathbb{R}^{d}} \psi(x) \phi\left(e^{t C} x+Z_{t}\right) d x \\
& =\left|\operatorname{det}\left(e^{-t C}\right)\right| \int_{\mathbb{R}^{d}} \psi\left(e^{-t C}\left(z-Z_{t}\right)\right) \phi(z) d z,\left(\operatorname{putting} z=e^{t C} x+Z_{t}\right) \\
& =e^{-t \operatorname{tr}(C)} \int_{\mathbb{R}^{d}}\left(\tau_{Z_{t}} \psi_{t}\right)(z) \phi(z) d z .
\end{aligned}
$$

The equality $\left|\operatorname{det}\left(e^{-t C}\right)\right|=e^{-t \operatorname{tr}(C)}$ follows from [8, Problem 5.6.P43]. Hence $Y_{t}(\psi)=e^{-t \operatorname{tr}(C)} \tau_{Z_{t}} \psi_{t}(\cdot)$.

Remark 3.12. We obtained probabilistic representation of solutions of (1.2), when the initial condition $\psi \in \mathcal{L}^{1}\left(\mathbb{R}^{d}\right)$ and the coefficients $\sigma, b$ are in a specific form. Possible extensions of these results to the case - when the initial condition $\psi \in \mathcal{L}^{q}\left(\mathbb{R}^{d}\right)$ for some $q>1$ or more generally a finite linear combination of the distributional derivatives of $\mathcal{L}^{q}\left(\mathbb{R}^{d}\right)$ functions $(q \geq 1)$ - will be taken up as future work.

Acknowledgement: The author would like to thank Professor B. Rajeev, Indian Statistical Institute, Bangalore for valuable suggestions during the work.

\section{REFERENCES}

[1] Peter Baxendale and Theodore E. Harris, Isotropic stochastic flows, Ann. Probab. 14 (1986), no. 4, 1155-1179. MR 866340 (88c:60030)

[2] S. Bhar and B. Rajeev, Differential Operators on Hermite Sobolev Spaces, to appear in Proc. Indian Acad. Sci. Math. Sci..

[3] Jean-Michel Bismut, A generalized formula of Itô and some other properties of stochastic flows, Z. Wahrsch. Verw. Gebiete 55 (1981), no. 3, 331-350. MR 608026 (82e:60093)

[4] Vivek S. Borkar, Evolution of interacting particles in a Brownian medium, Stochastics 14 (1984), no. 1, 33-79. MR 774584 (86f:60073)

[5] Andrew Carverhill, Flows of stochastic dynamical systems: ergodic theory, Stochastics 14 (1985), no. 4, 273-317. MR 805125 (87c:58059)

[6] K. D. Elworthy, Stochastic dynamical systems and their flows, Stochastic analysis (Proc. Internat. Conf., Northwestern Univ., Evanston, Ill., 1978), Academic Press, New York-London, 1978, pp. 79-95. MR 517235 (80e:60074)

[7] Takeyuki Hida, Brownian motion, Applications of Mathematics, vol. 11, Springer-Verlag, New York, 1980, Translated from the Japanese by the author and T. P. Speed. MR 562914 (81a:60089)

[8] Roger A. Horn and Charles R. Johnson, Matrix analysis, second ed., Cambridge University Press, Cambridge, 2013. MR 2978290

[9] Nobuyuki Ikeda and Shinzo Watanabe, Stochastic flows of diffeomorphisms, Stochastic analysis and applications, Adv. Probab. Related Topics, vol. 7, Dekker, New York, 1984, pp. 179198. MR 776981 (86h:58144)

[10] Kiyosi Itō, Foundations of stochastic differential equations in infinite-dimensional spaces, CBMS-NSF Regional Conference Series in Applied Mathematics, vol. 47, Society for Industrial and Applied Mathematics (SIAM), Philadelphia, PA, 1984. MR 771478 (87a:60068)

[11] Jean Jacod and Albert N. Shiryaev, Limit theorems for stochastic processes, second ed., Grundlehren der Mathematischen Wissenschaften [Fundamental Principles of Mathematical Sciences], vol. 288, Springer-Verlag, Berlin, 2003. MR 1943877 (2003j:60001)

[12] H. Kunita, Stochastic differential equations and stochastic flows of diffeomorphisms, École d'été de probabilités de Saint-Flour, XII-1982, Lecture Notes in Math., vol. 1097, Springer, Berlin, 1984, pp. 143-303. MR 876080 (87m:60127)

[13] Hiroshi Kunita, Stochastic differential equations and stochastic flows of homeomorphisms, Stochastic analysis and applications, Adv. Probab. Related Topics, vol. 7, Dekker, New York, 1984, pp. 269-291. MR 776984 (86g:58145) 
[14] Stochastic flows and stochastic differential equations, Cambridge Studies in Advanced Mathematics, vol. 24, Cambridge University Press, Cambridge, 1997, Reprint of the 1990 original. MR 1472487 (98e:60096)

[15] Paul Malliavin, Géométrie différentielle stochastique, Séminaire de Mathématiques Supérieures [Seminar on Higher Mathematics], vol. 64, Presses de l'Université de Montréal, Montreal, Que., 1978, Notes prepared by Danièle Dehen and Dominique Michel. MR 540035 (81d:60077)

[16] P.-A. Meyer, Flot d'une équation différentielle stochastique (d'après Malliavin, Bismut, Kunita), Seminar on Probability, XV (Univ. Strasbourg, Strasbourg, 1979/1980) (French), Lecture Notes in Math., vol. 850, Springer, Berlin-New York, 1981, pp. 103-117. MR 622556 (84c:60083a)

[17] Philip E. Protter, Stochastic integration and differential equations, second ed., Applications of Mathematics (New York), vol. 21, Springer-Verlag, Berlin, 2004, Stochastic Modelling and Applied Probability. MR 2020294 (2005k:60008)

[18] B. Rajeev, Translation invariant diffusions in the space of tempered distributions, Indian J. Pure Appl. Math. 44 (2013), no. 2, 231-258. MR 3063763

[19] B. Rajeev and S. Thangavelu, Probabilistic representations of solutions of the forward equations, Potential Anal. 28 (2008), no. 2, 139-162. MR 2373102 (2009e:60139)

[20] Daniel Revuz and Marc Yor, Continuous martingales and Brownian motion, third ed., Grundlehren der Mathematischen Wissenschaften [Fundamental Principles of Mathematical Sciences], vol. 293, Springer-Verlag, Berlin, 1999. MR 1725357 (2000h:60050)

[21] Daniel W. Stroock, Lectures on topics in stochastic differential equations, Tata Institute of Fundamental Research Lectures on Mathematics and Physics, vol. 68, Tata Institute of Fundamental Research, Bombay; by Springer-Verlag, Berlin-New York, 1982, With notes by Satyajit Karmakar. MR 685758 (84m:60071)

[22] H. van Bargen and G. Dimitroff, Isotropic Ornstein-Uhlenbeck flows, Stochastic Process. Appl. 119 (2009), no. 7, 2166-2197. MR 2531088 (2010g:60106)

[23] Shinzo Watanabe, Stochastic flows of diffeomorphisms, Probability theory and mathematical statistics (Tbilisi, 1982), Lecture Notes in Math., vol. 1021, Springer, Berlin, 1983, pp. 699708. MR 736036 (85h:60090)

Suprio Bhar, Indian Statistical Institute Bangalore Centre.

E-mail address: suprio@isibang.ac.in 